\title{
Analytical Determination of the Vibrations of a Load, Twice Elastically Suspended From an Elastic Beam via Another Load
}

\author{
Vassil Zlatanov $^{1}$, Stefan Bachvarov ${ }^{2} \&$ Snezhana Atanasova $^{1}$ \\ ${ }^{1}$ Dept. of Technical Mechanics, University of Food Technologies, Plovdiv, Bulgaria \\ ${ }^{2}$ Dept. of Mechanics, Technical University, Sofia, Bulgaria \\ Correspondence: Vassil Zlatanov, Dept. of Technical Mechanics, University of Food Technologies, Maritza 26, \\ Plovdiv 4002, Bulgaria. Tel: 359-3260-3827. E-mail: vassilzlatanov@mail.bg
}

Snezhana Atanasova, Dept. of Technical Mechanics, University of Food Technologies, Maritza 26, Plovdiv 4002, Bulgaria. Tel: 359-3260-3823. E-mail: sneja_atan@yahoo.com

$\begin{array}{lc}\text { Received: July 14, } 2014 & \text { Accepted: July 29, } 2014 \quad \text { Online Published: September 17, } 2014 \\ \text { doi:10.5539/mer.v4n2p30 } & \text { URL: http://dx.doi.org/10.5539/mer.v4n2p30 }\end{array}$

\begin{abstract}
This paper analyzes the dynamics of mechanical systems with suspended loads, such as bridge cranes, monorail hoists, mining machinery, etc. The considered mechanical system is composed of a load, twice elastically suspended from an elastic beam via another load. Two dynamical models which respect the kinetic energy of the elastic ropes are built for the system and their corresponding differential equations of motion are obtained. The vibration of the mechanical system is described by a coupled system of two ordinary and $n(n=1,2, \ldots)$ partial differential equations. The nonlinear restoring forces are linearized via the method of equivalent linearization and an analytical solution is obtained for the differential equations of both dynamical models simultaneously, using general initial conditions. The constants of integration are determined analytically for a specific instance of the initial conditions, which reflects an important practical case. The mechanical system is simulated numerically with initial conditions corresponding to the typical regimes of operation of real systems with suspended loads.
\end{abstract}

Keywords: analytical methods, suspended loads, vibration, dynamic model, elastic beam

\section{Introduction}

Mechanical systems with suspended loads, such as bridge cranes, monorail hoists, mining machinery, etc., are widely used for automating the transportation process in various sectors of the production industry. These systems possess interesting and diverse dynamic prosperities, the analysis of which is crucial for the improvement of existing and the introduction of new equipment.

The dynamics at points of suspension of elastic beams are analyzed in the classical monographs (Den Hartog, 1956, Timoshenko, Young, \& Weaver, 1974; Panavko, 1976, De Silva \& Clarence, 2000; Lurie, 2001). Fryba (1999), Timoshenko et al. (1974) present a solution for the vibration of a simply supported elastic beam caused by moving loads. A beam model with irregularities, subjected to a moving system with two degrees of freedom is presented by Fryba (1999). The model is analyzed using Runge-Kutta-Nystrom's method. Andrianov, Danishevs'ky, and Ivankov (2010) presented asymptotic methods for natural, free, and forced oscillations of beams and plates. The movement of a mechanical system with a suspended load is modeled as a mathematical pendulum with a movable suspension point and is analyzed via the method of Krylov-Bogolubov in (Bojaddjiev \& Butschvarov, 1967; Butschvarov, 1970). Analytical methods for solving the differential equations of motion of the mechanical systems in question are presented in (Kostin \& Saurin, 2007; Ouyang \& Mottershead 2007; Martikka \& Pollanen, 2014). The advantage of exact and approximate analytical methods: Lindstedt-Poincare method, the method of harmonic balance, methods of averaging, the method of multiples scales etc. is that we have exact and reliable result of the solution of a task. The method of finite elements has been used to study the vibration of elastic beams numerically and experimentally in several related works (Taylor, Fillipou, Saritas, \& Arricchio, 2003; Zvang \& Zheng, 2010; Popov, 2012; Prokic, Besevic, \& Lukic, 2014). Application of numerical methods leads to receiving simply result but it is still necessary to prove its reliability and to estimate error size.

Our previous work (Zlatanov, Buchvarov, Atanasova, 2012) presents a dynamical model of a mechanical system with a load suspended from a simply supported elastic beam via another load. In this paper we present a 
mechanical system composed of a load, twice elastically suspended from an elastic beam via another load. The goal is to determine the vibrations of the mechanical system with suspended loads. We use two dynamical models to analyze the dynamic load on the elastic ropes and the beam at various displacements from the suspension point.

\section{Modeling Mechanical Systems With Suspended Loads}

Designing robust and reliable suspended load systems requires an accurate determination of the dynamic loads acting on the different elements of the system. Consequently, developing a high-fidelity mechanical-mathematical model is critical for addressing this important engineering problem.

\subsection{Dynamical Models}

In this paper, bridge cranes, monorail hoists, and other mechanical systems with suspended loads are represented using two dynamical models which are shown in Figure 1 and Figure 2. These models take into account the system's characteristics and are based on the following assumptions:

1) The beam is regarded as a body with uniformly distributed mass made of a homogeneous, viscoelastic (Kelvin-Voigt type) material. The beam has length, $L$; flexural rigidity, $E J$; mass per unit length, $\mu$; viscoelastic parameter- $k_{0}$. It is supported on two rigid supports A and B and its displacement is assumed sufficiently small.

2) A point mass $m$ is rigidly attached to the beam at distance $a$ from support point A;

3) The first load is modeled as a point mass $m_{2}^{\prime}$ and is suspended via an elastic rope. The rope is attached to mass $m$ via a hinge joint. The rope is modeled as a nonlinear spring with stiffness $c_{2}, c_{4}\left(c_{4}=\gamma_{2} c_{2}, \gamma_{2}<1\right)$, coefficient of viscous damping $c_{a}$, free length $l_{0,2}$ and linear density $p_{2}$. To take into account the kinetic energy of the rope, we determine the combined mass $m_{2}$ according to the following expression (Stepanov, 1999): if $\alpha_{2}=\frac{l_{0,2} p_{2}}{m_{2}^{\prime}}$ and $0<\alpha_{2}<1$ then $m_{2}=m_{2}^{\prime}+\left(0.333+0.019 \alpha_{2}\right) l_{0,2} p_{2}$, or if $1<\alpha_{2}<2$ then $m_{2}=m_{2}^{\prime}+\left[0.351+0.100\left(\alpha_{2}-1\right)\right] l_{0,2} p_{2}$

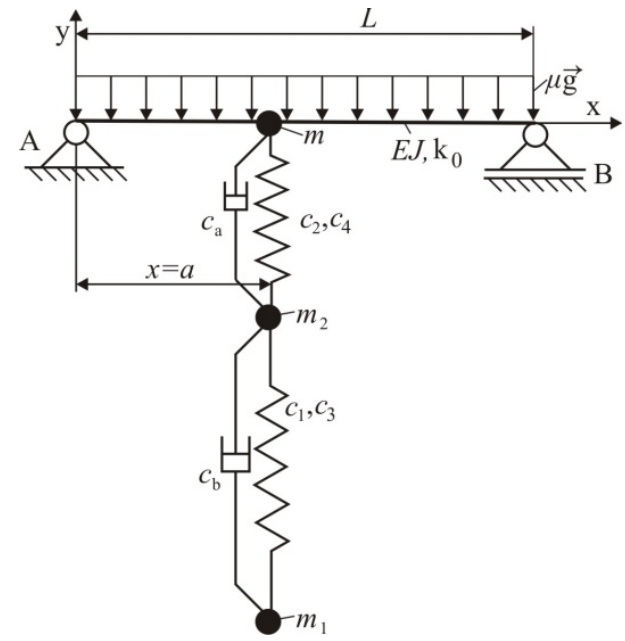

Figure 1. Dynamic model 1

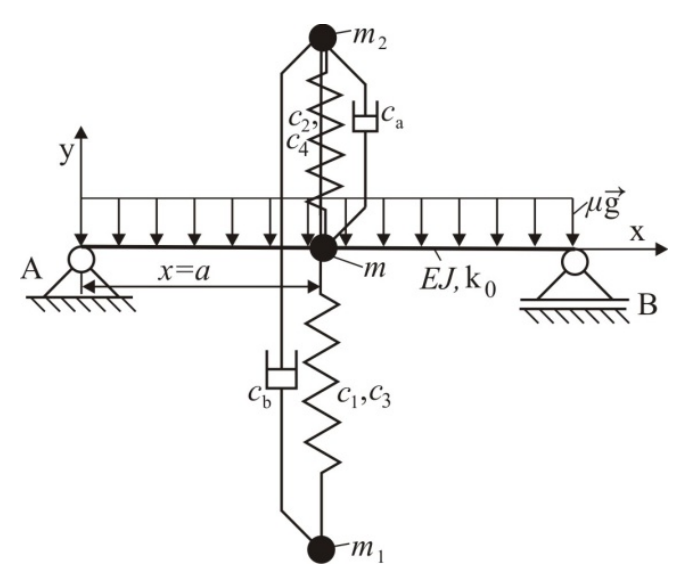

Figure 2. Dymamic model 2

4) The suspended load is modeled as a point mass $m_{1}^{\prime}$ suspended via an elastic rope and a hinge joint. The rope is modeled as a non-linear spring with stiffness $c_{1}, c_{3}\left(c_{3}=\gamma_{1} c_{1}, \gamma_{1}<1\right)$, coefficient of viscous damping $c_{b}$, free length $l_{0,1}$ and linear density $p_{1}$. To take into account the kinetic energy of the rope, the combined point mass $m_{1}$ is determined according to the following expression (Stepanov, 1999): $\alpha_{1}=\frac{l_{0,1} p_{1}}{m_{1}^{\prime}}$ and $0<\alpha_{1}<1: m_{1}=m_{1}^{\prime}+\left(0.333+0.019 \alpha_{1}\right) l_{0,1} p_{1}$, or if $1<\alpha_{1}<2: m_{1}=m_{1}^{\prime}+\left[0.351+0.100\left(\alpha_{1}-1\right)\right] l_{0,1} p_{1}$. 
5) It is assumed that without deformation the elastic axis of the beam is a straight line, which coincides with the centers of mass of the cross sections. Let $\mathrm{x}$ denote this axis. It will also be assumed that the displacements of the individual points from the axis of the beam are perpendicular to its undistorted state, i.e. the deviations parallel to the axis will be ignored because they are second order quantities, which are negligibly small compared to the transverse displacements. The displacements of the individual points from the axis are confined to a single plane and are small in the sense that the restoring forces remain proportional to them.

\subsection{Differential Equations of Motion}

Beam models are shown in Figure 3 and Figure 4.

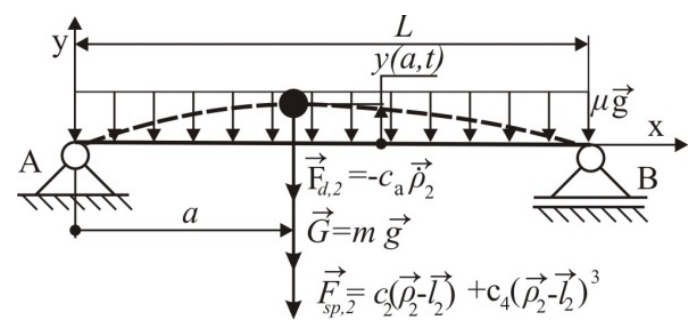

Figure 3. Beam of system 1

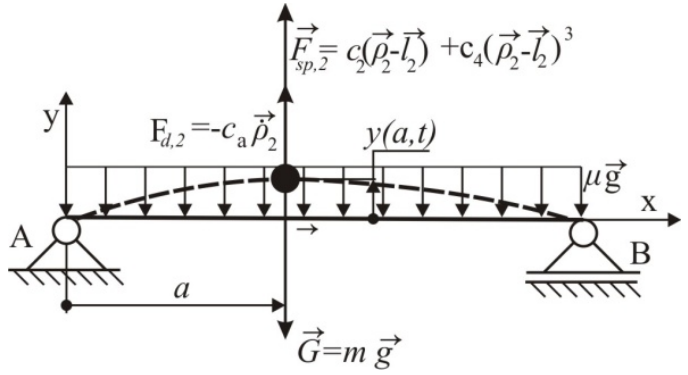

Figure 4. Beam of system 2

The Bernoulli-Euler equation of bending motion of a beam with viscoelastic material damping subjected to a load of $q(x, t)$ is given by Panavko, (1976):

$$
\mu \frac{\partial^{2} y}{\partial t^{2}}+k_{0} J \frac{\partial^{5} y}{\partial t \partial x^{4}}+E J \frac{\partial^{4} y}{\partial x^{4}}=q(x, t) .
$$

Here, $y=y(x, t)$ is the transverse motion at a distance $x$ along the beam.

Vibrating systems are shown in Figure 5 and Figure 6. The vibrating system 1 and system 2 have two degrees of freedom and the suspension points are movable. The differential equations of the small displacements can be obtained using the method of Lagrange, direct or inverse method (Panavko, 1976). We have used the direct method.

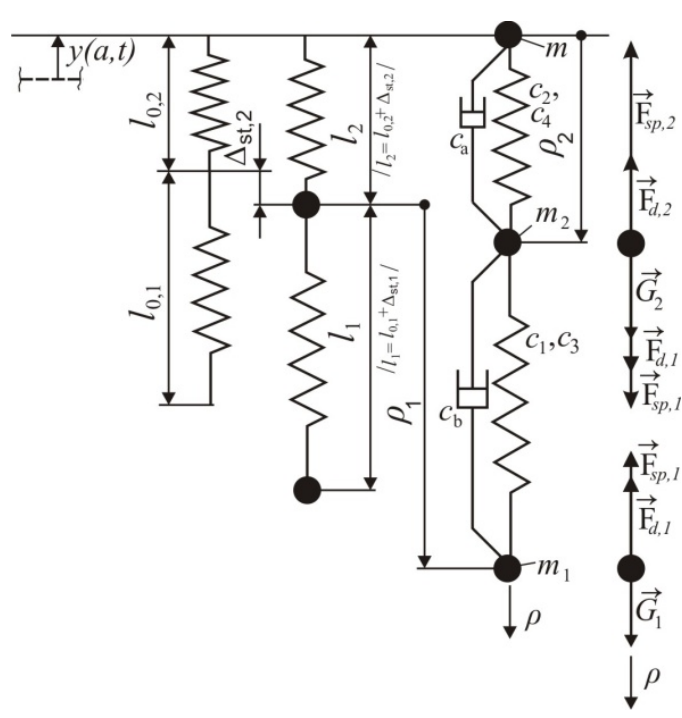

Figure 5. Vibrating system 1

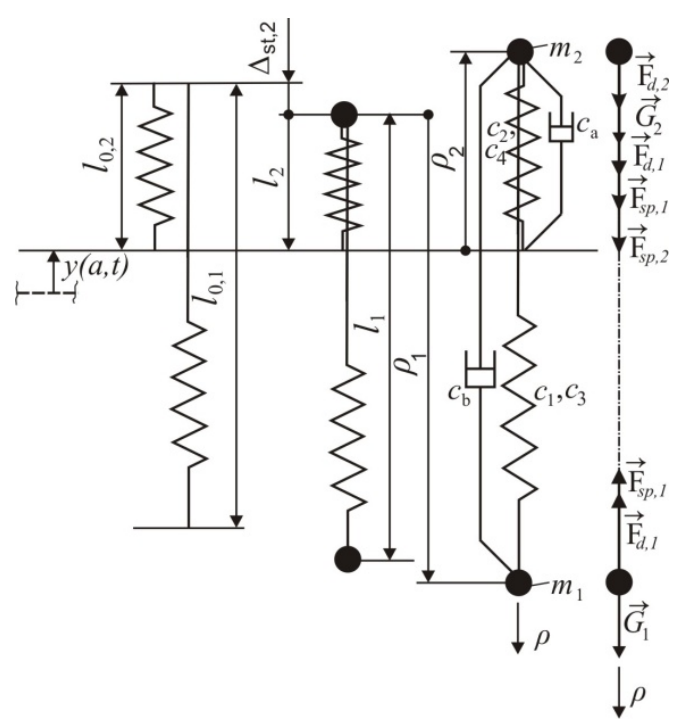

Figure 6. Vibrating system 2

The mass $m_{1}$ in the vibrating system in dynamical model 1 is acted upon by the dynamic components of the elastic force $\vec{F}_{s p, 1}$ and the viscous drag force $\vec{F}_{d, 1}$. The equation of absolute motion of mass $m_{1}$ relative to the coordinate system Axy has the form: 


$$
m_{1} \ddot{\rho}_{1}-m_{1} \ddot{y}=-c_{1}\left[\left(\rho_{1}-l_{1}\right)-\left(\rho_{2}-l_{2}\right)\right]-c_{3}\left[\left(\rho_{1}-l_{1}\right)-\left(\rho_{2}-l_{2}\right)\right]^{3}-c_{b}\left(\dot{\rho}_{1}-\dot{\rho}_{2}\right) .
$$

where $l_{1}$ and $l_{2}$ denote the lengths of the springs in equilibrium, while $\rho_{1}$ and $\rho_{2}$-their current lengths.

The mass $m_{2}$ is acted upon by the dynamic components of the elastic forces $\vec{F}_{s p, 1}, \vec{F}_{s p, 2}$ and the viscous drag forces $\vec{F}_{d, 1}, \vec{F}_{d, 2}$. The equation of absolute motion of mass $m_{2}$ has the form:

$$
\begin{aligned}
m_{2} \ddot{\rho}_{1}-m_{2} \ddot{y} & =c_{1}\left[\left(\rho_{1}-l_{1}\right)-\left(\rho_{2}-l_{2}\right)\right]+c_{3}\left[\left(\rho_{1}-l_{1}\right)-\left(\rho_{2}-l_{2}\right)\right]^{3}+ \\
& +c_{b}\left(\dot{\rho}_{1}-\dot{\rho}_{2}\right)-c_{2}\left(\rho_{2}-l_{2}\right)-c_{4}\left(\rho_{2}-l_{2}\right)^{3}-c_{a} \dot{\rho}_{2} .
\end{aligned}
$$

Analogously, we arrive at the following differential equations for the absolute motion of the masses $m_{1}$ and $m_{2}$ in dynamical model 2:

$$
\begin{gathered}
m_{1} \ddot{\rho}_{1}-m_{1} \ddot{y}=-c_{1}\left[\left(\rho_{1}-l_{1}\right)+\left(\rho_{2}-l_{2}\right)\right]-c_{3}\left[\left(\rho_{1}-l_{1}\right)+\left(\rho_{2}-l_{2}\right)\right]^{3}-c_{b}\left(\dot{\rho}_{1}+\dot{\rho}_{2}\right), \\
-m_{2} \ddot{\rho}_{1}-m_{2} \ddot{y}=c_{1}\left[\left(\rho_{1}-l_{1}\right)+\left(\rho_{2}-l_{2}\right)\right]+c_{3}\left[\left(\rho_{1}-l_{1}\right)+\left(\rho_{2}-l_{2}\right)\right]^{3}+ \\
+c_{b}\left(\dot{\rho}_{1}+\dot{\rho}_{2}\right)+c_{2}\left(\rho_{2}-l_{2}\right)+c_{4}\left(\rho_{2}-l_{2}\right)^{3}+c_{a} \dot{\rho}_{2} .
\end{gathered}
$$

Letting $\eta_{1}(t)=\rho_{1}(t)-l_{1}$ and $\eta_{2}(t)=\rho_{2}(t)-l_{2}$ leads to the following system of differential equations of motion:

$$
\begin{aligned}
& \mu \frac{\partial^{2} y}{\partial t^{2}}+k_{0} J \frac{\partial^{5} y}{\partial t \partial x^{4}}+E J \frac{\partial^{4} y}{\partial x^{4}}=q(x, t), \\
& m_{1} \ddot{\eta}_{1}=m_{1} \ddot{y}-c_{1}\left(\eta_{1}-\eta_{2}\right)-c_{3}\left(\eta_{1}-\eta_{2}\right)^{3}-c_{b}\left(\dot{\eta}_{1}-\dot{\eta}_{2}\right), \\
& m_{2} \ddot{\eta}_{2}=m_{2} \ddot{y}+c_{1}\left(\eta_{1}-\eta_{2}\right)+c_{3}\left(\eta_{1}-\eta_{2}\right)^{3}+c_{b}\left(\dot{\eta}_{1}-\dot{\eta}_{2}\right)-c_{2} \eta_{2}-c_{4} \eta_{2}{ }^{3}-c_{a} \dot{\eta}_{2} .
\end{aligned}
$$

The differential equations for dynamical model 2 have the form:

$$
\begin{aligned}
& \mu \frac{\partial^{2} y}{\partial t^{2}}+k_{0} J \frac{\partial^{5} y}{\partial t \partial x^{4}}+E J \frac{\partial^{4} y}{\partial x^{4}}=q(x, t), \\
& m_{1} \ddot{\eta}_{1}=m_{1} \ddot{y}-c_{1}\left(\eta_{1}+\eta_{2}\right)-c_{3}\left(\eta_{1}+\eta_{2}\right)^{3}-c_{b}\left(\dot{\eta}_{1}+\dot{\eta}_{2}\right), \\
& m_{2} \ddot{\eta}_{2}=-m_{2} \ddot{y}-c_{1}\left(\eta_{1}+\eta_{2}\right)-c_{3}\left(\eta_{1}+\eta_{2}\right)^{3}-c_{b}\left(\dot{\eta}_{1}+\dot{\eta}_{2}\right)-c_{2} \eta_{2}-c_{4} \eta_{2}{ }^{3}-c_{a} \dot{\eta}_{2} .
\end{aligned}
$$

The boundary conditions are:

$$
y(0, t)=0,\left.\frac{\partial^{2} y(x, t)}{\partial x^{2}}\right|_{x=0}=0, \quad y(l, t)=0,\left.\quad \frac{\partial^{2} y(x, t)}{\partial x^{2}}\right|_{x=l}=0
$$

and the initial conditions are:

$$
\begin{aligned}
& \eta_{1}(0)=\eta_{1,0}=\rho_{1}(0)-l_{1}, \dot{\eta}_{1}(0)=\dot{\eta}_{1,0}=\dot{\rho}_{1}(0), \eta_{2}(0)=\eta_{2,0}=\rho_{2}(0)-l_{2}, \dot{\eta}_{2}(0)=\dot{\eta}_{2,0}=\dot{\rho}_{2}(0), \\
& y(x, 0)=y_{0}(x),\left.\frac{\partial y(x, t)}{\partial t}\right|_{t=0}=\dot{y}_{0}(x) .
\end{aligned}
$$

\section{Solving the System of Differential Equations}

To solve the system of three coupled equations in Equation (6) or Equation (7) we follow a two-step approach.

\subsection{First Step}

In this work, we replace the elastic nonlinear forces $F_{s p, 1}=f_{1}\left(\eta_{1}\right)=c_{1} \eta_{1}+c_{3} \eta_{1}{ }^{3}$ and $F_{s p, 2}=f_{2}\left(\eta_{2}\right)=c_{2} \eta_{2}+c_{4} \eta_{2}{ }^{3}$ with corresponding linearized elastic forces $F_{s p, 1}^{*}=f_{1}^{s *}\left(\eta_{1}\right)=c_{1}^{*} \eta_{1}$ and $F_{s p, 2}^{*}=f_{2}^{*}\left(\eta_{2}\right)=c_{2}^{*} \eta_{2}$, determined using direct linearization (Buchvarov, Tcherneva-Popova, \& Banov 1998). The coefficients $c_{i}^{*}, i=1,2$ are obtained by minimizing the following integral: 


$$
I\left(c_{i}^{*}\right)=\int_{-A_{m, i}}^{A_{m, i}}\left\{\left[f_{i}\left(\eta_{i}\right)-c_{i}^{*} \eta_{i}\right] \lambda\left(\eta_{i}\right)\right\}^{2} d \eta_{i} \quad, i=1,2,
$$

where $f_{i}\left(\eta_{i}\right)-c_{i}^{*} \eta_{i}$ is the deviation of the elastic force from the linearization in the range $\left[-A_{m, i}, A_{m, i}\right]$, determined by the amplitude $A_{m, i}$. We assume that the importance (weight) of the deviation is proportional to the x-axis, i.e. $\lambda\left(\eta_{i}\right)=\eta_{i}$ and we find:

$$
c_{1}^{*}=c_{1}+\frac{5}{7} c_{3} A_{m, 1}^{2}, \quad c_{2}^{*}=c_{2}+\frac{5}{7} c_{4} A_{m, 2}{ }^{2} .
$$

The vibration amplitudes $A_{m, i}$ will be determined using the method in Zlatanov, Buchvarov, \& Atanasova (2012). In null approximation, we assume that the amplitudes $A_{m, i}$ are equal to the amplitudes of free vibrations of the system, which result from the particular initial conditions. We represent the systems of ordinary differential equations from Equation (6) and Equation (7) in the following form:

$$
\begin{aligned}
& a_{11} \ddot{\eta}_{1}+a_{12} \ddot{\eta}_{2}+b_{11} \dot{\eta}_{1}+b_{12} \dot{\eta}_{2}+c_{11} \eta_{1}+c_{12} \eta_{2}=Q_{1}(t), \\
& a_{21} \ddot{\eta}_{1}+a_{22} \ddot{\eta}_{2}+b_{21} \dot{\eta}_{1}+b_{22} \dot{\eta}_{2}+c_{21} \eta_{1}+c_{22} \eta_{2}=Q_{2}(t),
\end{aligned}
$$

where for dynamical model 1: $\begin{aligned} & a_{11}=m_{1}, a_{12}=a_{21}=0, c_{11}=c_{1}^{*}, c_{12}=c_{21}=-c_{1}^{*}, b_{11}=c_{b}, b_{12}=b_{21}=-c_{b}, \\ & a_{22}=m_{2}, c_{22}=c_{1}{ }^{*}+c_{2}{ }^{*}, b_{22}=c_{a}+c_{b}, Q_{1}=m_{1} \ddot{y}(a, t), Q_{2}=m_{2} \ddot{y}(a, t),\end{aligned}$

and for dynamical model 2: $a_{11}=m_{1}, a_{12}=a_{21}=0, c_{11}=c_{1}^{*}, c_{12}=c_{21}=c_{1}^{*}, b_{11}=c_{b}, b_{12}=b_{21}=c_{b}$,

$$
a_{22}=m_{2}, c_{22}=c_{1}^{*}+c_{2}^{*}, b_{22}=c_{a}+c_{b}, Q_{1}=m_{1} \ddot{y}(a, t), Q_{2}=-m_{2} \ddot{y}(a, t) \text {. }
$$

The free vibrations of the point masses $m_{1}$ and $m_{2}$ are determined by solving the following homogeneous system:

$$
\begin{aligned}
& a_{11} \ddot{\eta}_{1}+c_{11} \eta_{1}+c_{12} \eta_{2}=0, \\
& a_{22} \ddot{\eta}_{2}+c_{21} \eta_{1}+c_{22} \eta_{2}=0 .
\end{aligned}
$$

System in Equation (13) is obtained from Equation (12) by neglecting the viscous drag and in zeroth-order approximation assuming $c_{1}^{*}=c_{1}, c_{2}{ }^{*}=c_{2}, m_{i} \ddot{y}(a, 0)=0$. The solution of system from Equation (13) is known to have the form (Ilin, Kolesnikov, \& Saratov, 2001):

$$
\begin{aligned}
& \eta_{1}=A_{11} \sin \left(\omega_{1}^{*} t+\alpha_{1}\right)+A_{12} \sin \left(\omega_{2}^{*} t+\alpha_{2}\right), \\
& \eta_{2}=\mu_{21} A_{11} \sin \left(\omega_{1}^{*} t+\alpha_{1}\right)+\mu_{22} A_{12} \sin \left(\omega_{2}^{*} t+\alpha_{2}\right),
\end{aligned}
$$

where $\mu_{21}=-\frac{c_{11}-\omega_{1}^{* 2} a_{11}}{c_{12}}, \mu_{22}=-\frac{c_{11}-\omega_{2}^{* 2} a_{11}}{c_{12}}$, and the squares of the natural frequencies are:

$$
\omega_{1,2}^{*}=\frac{c_{11} a_{22}+c_{22} a_{11} \pm \sqrt{\left(c_{11} a_{22}+c_{22} a_{11}\right)^{2}-4 a_{11} a_{22}\left(c_{11} c_{22}-c_{12}\right)^{2}}}{2 a_{11} a_{22}} .
$$

The solution contains four undetermined coefficients: $A_{11}, A_{12}, \alpha_{1}, \alpha_{2}$. With general initial conditions in Equation (9), the coefficients $A_{11}, A_{12}, \alpha_{1}, \alpha_{2}$ can be obtained from the following system:

$$
\begin{aligned}
& \eta_{1,0}=A_{11} \sin \alpha_{1}+A_{12} \sin \alpha_{2}, \quad \dot{\eta}_{1,0}=\omega_{1}^{*} A_{11} \cos \alpha_{1}+\omega_{2}^{*} A_{12} \cos \alpha_{2}, \\
& \eta_{2,0}=\mu_{21} A_{11} \sin \alpha_{1}+\mu_{22} A_{12} \sin \alpha_{2}, \dot{\eta}_{1,0}=\mu_{21} \omega_{1}^{*} A_{11} \cos \alpha_{1}+\mu_{22} \omega_{2}^{*} A_{12} \cos \alpha_{2} .
\end{aligned}
$$

We derive $A_{11}, A_{12}, \alpha_{1}, \alpha_{2}$ for initial conditions with:

$$
\eta_{1,0}=0, \dot{\eta}_{1,0} \neq 0, \eta_{2,0}=0, \dot{\eta}_{2,0}=0 .
$$

These initial conditions correspond to a common regime of operation in the real mechanical system, namely when the rope is descending (ascending) with a constant linear velocity and its motion is suddenly stopped. Since $A_{11}$ and $A_{12}$ are nonzero, the first and third equations from Equation (16), with initial conditions in Equation (17), 
imply that $\sin \alpha_{1}=0$ and $\sin \alpha_{2}=0$ and in turn $\alpha_{1}=0, \pi, 2 \pi, \ldots$ and $\alpha_{2}=0, \pi, 2 \pi, \ldots$. As $A_{11}$ and $A_{12}$ are undetermined, we can choose any value for $\alpha_{1}$ and $\alpha_{2}$. From the second and fourth equations in Equation (16) with $\alpha_{1}=0$ and $\alpha_{2}=0$, we obtain:

$$
A_{11}=\frac{\dot{\eta}_{1,0}}{\omega_{1}^{*}} \frac{\mu_{22}}{\mu_{22}-\mu_{21}}, A_{12}=-\frac{\dot{\eta}_{1,0}}{\omega_{2}^{*}} \frac{\mu_{21}}{\mu_{22}-\mu_{21}} .
$$

These results enable us to determine $A_{m, 1}$ and $A_{m, 2}$ for the zero approximation, and in turn via Equation (11) the coefficients $c_{1}^{*}$ and $c_{2}^{*}$.

We consider the following system of differential equations, in order to obtain the standing equilibrium displacement of the springs with stiffness $c_{1}^{*}$ and $c_{2}{ }^{*}$ :

$$
\begin{aligned}
& a_{11} \ddot{\eta}_{1}+c_{11} \eta_{1}+c_{12} \eta_{2}=F_{1}^{*} \sin \left(\omega^{*} t\right), \\
& a_{22} \ddot{\eta}_{2}+c_{21} \eta_{1}+c_{22} \eta_{2}=F_{2}^{*} \sin \left(\omega^{*} t\right) .
\end{aligned}
$$

If we let $\omega^{*}=0$ and $F_{1}^{*}=m_{1} g, F_{2}^{*}=m_{2} g$ for dynamical model 1 and $F_{1}^{*}=m_{1} g, F_{2}^{*}=-m_{2} g$ for dynamical model 2, then the expressions for $\eta_{1}, \eta_{2}\left(\eta_{1}=\rho_{1}-l_{0,1}, \eta_{2}=\rho_{2}-l_{0,2}\right)$ obtained from Equation (19) will correspond to the standing equilibrium displacement of the spring. We suppose that the solution has the form:

$$
\eta_{1}=A \sin \left(\omega^{*} t\right) \quad, \quad \eta_{2}=B \sin \left(\omega^{*} t\right)
$$

We substitute Equation (20) in Equation (19), rearrange, solve the resulting system of algebraic equations using Cramer's method, and obtain:

$$
A=\frac{F_{1}^{*}\left(-a_{22} \omega^{* 2}+c_{22}\right)-F_{2}^{*} c_{12}}{\left(-a_{11} \omega^{* 2}+c_{11}\right)\left(-a_{22} \omega^{* 2}+c_{22}\right)-c_{12} c_{21}}, B=\frac{F_{2}^{*}\left(-a_{11} \omega^{* 2}+c_{11}\right)-F_{1}^{*} c_{21}}{\left(-a_{11} \omega^{* 2}+c_{11}\right)\left(-a_{22} \omega^{* 2}+c_{22}\right)-c_{12} c_{21}}
$$

Letting $\omega^{*}=0$ in Equation (21), we get the following for the two dynamical models:

$$
\text { dynamical model } 1
$$

dynamical model 2

$$
\Delta_{s t, 1}=\left(m_{1} \frac{c_{1}{ }^{*}+c_{2}^{*}}{c_{1}{ }^{*} c_{2}{ }^{*}}+m_{2} \frac{1}{c_{2}^{*}}\right) g, \Delta_{s t, 2}=\frac{\left(m_{1}+m_{2}\right) g}{c_{2}{ }^{*}} ; \quad \Delta_{s t, 1}=\left(m_{1} \frac{c_{1}^{*}+c_{2}{ }^{*}}{c_{1}{ }^{*} c_{2}{ }^{*}}+m_{2} \frac{1}{c_{2}{ }^{*}}\right) g, \Delta_{s t, 2}=-\frac{\left(m_{1}+m_{2}\right) g}{c_{2}{ }^{*}} ;
$$

At this stage, we assume that the acceleration of the points in the beam section with $x=a$ coincides with their acceleration at time $t=0, \quad m_{1} \ddot{y}(a, t)=\left.m_{1} \frac{\partial^{2} y(x, t)}{\partial t^{2}}\right|_{\substack{t=0 \\ x=a}}=$ const. Assuming that at zero deviation $\left.\frac{\partial^{2} y(x, t)}{\partial t^{2}}\right|_{\substack{t=0 \\ x=a}}=0$, we obtain Equation (12) in a form corresponding to the differential equations of small oscillations caused by conservative and dissipative forces:

$$
\begin{aligned}
& a_{11} \ddot{\eta}_{1}+a_{12} \ddot{\eta}_{2}+b_{11} \dot{\eta}_{1}+b_{12} \dot{\eta}_{2}+c_{11} \eta_{1}+c_{12} \eta_{2}=0, \\
& a_{21} \ddot{\eta}_{1}+a_{22} \ddot{\eta}_{2}+b_{21} \dot{\eta}_{1}+b_{22} \dot{\eta}_{2}+c_{21} \eta_{1}+c_{22} \eta_{2}=0,
\end{aligned}
$$

We seek a solution to Equation (22) in the following form:

$$
\eta_{1}=A_{1} e^{\lambda_{1} t} \quad, \quad \eta_{2}=A_{2} e^{\lambda_{2} t}
$$

where $A_{1}, A_{2}, \lambda$ are constants to be determined.

We substitute $\eta_{1}$ and $\eta_{2}$ from Equation (23) and their derivatives in Equation (22). The roots of the characteristic equation 


$$
\left|\begin{array}{ll}
a_{11} \lambda^{2}+b_{11} \lambda+c_{11} & a_{12} \lambda^{2}+b_{12} \lambda+c_{12} \\
a_{21} \lambda^{2}+b_{21} \lambda+c_{21} & a_{22} \lambda^{2}+b_{22} \lambda+c_{22}
\end{array}\right|=0
$$

have the form (Butenin, Lunts, \& Merkin, 1985): $\lambda=-h \pm \omega i, \lambda=-m, \lambda= \pm k i$, where $h, \omega, m, k$ are positive reals. For relatively small viscous damping forces, which are assumed in this work, the roots of the characteristic equation are complex conjugates with negative real parts: $-h_{1} \pm \omega_{1} i,-h_{2} \pm \omega_{2} i$. Thus, the solution of Equation (22) has the form:

$$
\begin{aligned}
& \eta_{1}=A_{11} e^{-h_{1} t} \sin \left(\omega_{1} t+\alpha_{1}\right)+A_{12} e^{-h_{2} t} \sin \left(\omega_{2} t+\alpha_{2}\right), \\
& \eta_{2}=A_{21} e^{-h_{1} t} \sin \left(\omega_{1} t+\alpha_{1}\right)+A_{22} e^{-h_{2} t} \sin \left(\omega_{2} t+\alpha_{2}\right) .
\end{aligned}
$$

We assume that the dissipative forces depend on a small parameter $\varepsilon$, i.e.

$$
b_{j k}=\varepsilon \beta_{j k} \quad, \quad(j=1,2 ; k=1,2)
$$

where $\beta_{j k}$ are finite numbers.

We substitute Equation (26) in Equation (24), expand the characteristic equation, and keep only the first order terms containing $\varepsilon$, i.e. $\mathrm{O}(\varepsilon)$ :

$$
a_{0} \lambda^{4}+\varepsilon a_{1} \lambda^{3}+a_{2} \lambda^{2}+\varepsilon a_{3} \lambda+a_{4}=0
$$

where $a_{0}=a_{11} a_{12}, a_{1}=\beta_{22} a_{11}+\beta_{11} a_{22}, a_{2}=a_{11} c_{22}+a_{22} c_{11}, a_{3}=\beta_{11} c_{22}+\beta_{22} c_{11}-\beta_{12} c_{21}-\beta_{21} c_{12}, a_{4}=c_{11} c_{22}-c_{12} c_{21}$.

If we let $\varepsilon=0$ and $\lambda= \pm k i$ in Equation (27), we obtain exactly the equation for the frequencies of the free vibrations, i.e. $k_{j}=\omega_{j}^{*}$. Thus, we obtain the following good approximation for the roots $\lambda=-h \pm \omega i$ of the characteristic equation:

$$
\omega_{j} \approx k_{j}=\omega_{j}^{*}, h_{j}=\frac{1}{2} \frac{a_{1} \omega_{j}^{2}-a_{3}}{2 a_{0} \omega_{j}^{2}-a_{2}} \varepsilon \quad(j=1,2),
$$

The expression for $h_{j}$ in Equation (28) is reasonable in the case that $\omega_{j}$ is not a multiple root of Equation (27) when $\varepsilon=0$.

The complete solution to Equation (22) has the form:

$$
\begin{aligned}
& \eta_{1}=A_{11} e^{-h_{1} t} \sin \left(\omega_{1} t+\alpha_{1}\right)+A_{12} e^{-h_{2} t} \sin \left(\omega_{2} t+\alpha_{2}\right), \\
& \eta_{2}=\mu_{21} A_{11} e^{-h_{1} t} \sin \left(\omega_{1} t+\alpha_{1}\right)+\mu_{22} A_{12} e^{-h_{2} t} \sin \left(\omega_{2} t+\alpha_{2}\right),
\end{aligned}
$$

where $\mu_{2 j}=-\frac{c_{11}-\omega_{j}^{2} a_{11}}{c_{12}}(j=1,2)$, while with the initial conditions from Equation (17) and $\alpha_{1}=0, \alpha_{2}=0$, we have $A_{11}=\frac{\dot{\eta}_{1,0}}{\omega_{1}} \frac{\mu_{22}}{\mu_{22}-\mu_{21}}, A_{12}=-\frac{\dot{\eta}_{1,0}}{\omega_{2}} \frac{\mu_{21}}{\mu_{22}-\mu_{21}}$.

The partial differential equation from Equation (6) and Equation (7) in canonical form are:

$$
\frac{\partial^{2} y}{\partial t^{2}}+\frac{k_{0} J}{\mu} \frac{\partial^{5} y}{\partial t \partial x^{4}}+\frac{E J}{\mu} \frac{\partial^{4} y}{\partial x^{4}}=\frac{q(x, t)}{\mu} .
$$

Using a Dirac delta function and taking the coordinate system Axy (see Figure 3) into account, we can write the right-hand side of Equation (30) as follows:

$$
q(x, t)=-\delta(x-a) m g-\delta(x-a) c_{2}^{*}\left(\eta_{2}+\Delta_{s t, 2}\right)-\delta(x-a) c_{a} \dot{\eta}_{2}-\left.\delta(x-a) m \frac{\partial^{2} y(x, t)}{\partial t^{2}}\right|_{x=a}-\mu g .
$$


To solve Equation (30), we can use a Fourier transform (Fryba, 1999) or we can represent the solution using eigenfunctions $X_{n}(x),(n=1, \ldots, \infty)$ of the elastic beam with length $L$, beam stiffness $E J=$ const, and fixed point mass $m$ at a distance $a$ from the left edge of the beam (Panavko, 1976). Here, we use the second method, i.e. the solution of Equation (30) is represented in the form:

$$
y(x, t)=\sum_{n=1}^{\infty} X_{n}(x) \cdot \psi_{n}(t) .
$$

The eigenfunctions $X_{n}(x),(n=1, \ldots, \infty)$, which are independent of the viscous properties and the square of the natural frequency $\bar{p}_{n}$ of the elastic simply supported beam, are obtained (Zlatanov, Buchvarov, \& Atanasova, 2012) in the form:

$$
X_{n}(x)=\sqrt{\frac{2}{L}} \sin \left(\frac{\pi n}{L} x\right), \bar{p}_{n}{ }^{2}=p_{n}{ }^{2} \frac{1}{1+\frac{2}{L} \frac{m}{\mu} \sin ^{2}\left(\frac{\pi n}{L} a\right)}=\frac{\pi^{4} n^{4}}{L^{4}} \frac{E J}{\mu} \frac{1}{1+\frac{2}{L} \frac{m}{\mu} \sin ^{2}\left(\frac{\pi n}{L} a\right)}(n=1,2, \ldots, \infty) .(32)
$$

We represent the right side of Equation (6) and Equation (7) in expanded form:

$$
q(x, t)=\sum_{n=1}^{\infty} X_{n}(x) \cdot S_{n}(t) .
$$

We multiply both sides with $X_{n}(x)$, integrate the resulting expression along the length $L$ of the beam, take into account the orthogonality and the normalization of the eigenfunctions, and get:

$$
S_{n}(t)=\frac{\int_{0}^{L} q(x, t) X_{n}(x) d x}{\int_{0}^{L} X_{n}{ }^{2}(x) d x}=-\sqrt{\frac{2}{L}}\left[\frac{\mu g L}{\pi n}(1-\cos (\pi n))+\left(m g \pm c_{2}{ }^{*} \eta_{2} \pm c_{2}{ }^{*} \Delta_{s t, 2} \pm c_{a} \dot{\eta}_{2}\right) \sin \left(\frac{\pi n}{L} a\right)\right],
$$

where \pm is + for dynamical model 1 and - for dynamical model 2 .

The eigenfunction series representation of the solution leads to the following $n$ equations:

$$
\ddot{\psi}_{n}+2 \alpha_{n} \dot{\psi}_{n}+\bar{p}_{n}^{2} \psi_{n}=\frac{S_{n}}{\mu} \quad, \quad\left(2 \alpha_{n}=\frac{k_{0} \bar{p}_{n}^{2}}{\mu}\right)
$$

In this first step of the solution, we assume that the right-hand side of Equation (33) is a constant, determined by the value of the functions $S_{n}(t)$ at time $t=0$, i.e. $K_{n}{ }^{\prime}=\frac{S_{n}(0)}{\mu}$. If $\alpha_{n}>\bar{p}_{n}$, the system is overdamped, which is typical for real elastic beams, and the solution to Equation (33) is:

$$
\psi_{n}=\frac{K_{n}^{\prime}}{\bar{p}_{n}^{2}}+\frac{e^{-\alpha_{n} t}}{\bar{p}_{n}^{2}}\left(C_{1 n}^{\prime} e^{p_{n}^{*} t}+C_{2 n}^{\prime} e^{-p_{n}^{*} t}\right)
$$

where $p_{n}^{*}=\sqrt{\alpha_{n}^{2}-\bar{p}_{n}^{2}}$ and $C_{1 n}^{\prime}, C_{2 n}^{\prime}$ are integration constants.

Taking Equation (31), Equation (32), and Equation (34) into account, the beam vibration has the following form:

$$
y(x, t)=\sum_{n=1}^{\infty} X_{n}(x) \psi_{n}(t)=\sum_{n=1}^{\infty}\left[C_{0 n}+e^{-\alpha_{n} t}\left(C_{1 n}^{\prime} e^{p_{n}^{*} t}+C_{2 n}^{\prime} e^{-p_{n}^{*} t}\right)\right],
$$

where $C_{0 n}=\sqrt{\frac{2}{L}} K_{n}^{\prime} \frac{1}{\bar{p}_{n}^{2}} \sin \left(\frac{\pi n}{L} x\right)$

We multiply $\left.y(x, t)\right|_{t=0}$ and $\left.\frac{\partial y(x, t)}{\partial t}\right|_{t=0}$ from Equation (35) by $X_{n}(x)$, integrate over the entire length of the beam, take the orthogonality of the eigenfunctions and initial conditions from Equation (9) into account, and get: 


$$
\begin{aligned}
& C_{1 n}^{\prime}=\frac{1}{\sqrt{2 L} p_{n}^{*}} \sin \left(\frac{\pi n}{L} x\right)\left\{\left\{\left(\int_{0}^{L} y_{0}(x) X_{n}(x) d x-\frac{K_{n}^{\prime}}{\bar{p}_{n}{ }^{2}}\right)\left(p_{n}^{*}+\alpha_{n}\right)+\int_{0}^{L} \dot{y}_{0}(x) X_{n}(x) d x\right\},\right. \\
& C_{2 n}^{\prime}=\frac{1}{\sqrt{2 L} p_{n}^{*}} \sin \left(\frac{\pi n}{L} x\right)\left\{\left(\int_{0}^{L} y_{0}(x) X_{n}(x) d x-\frac{K_{n}^{\prime}}{\bar{p}_{n}^{2}}\right)\left(p_{n}^{*}-\alpha_{n}\right)-\int_{0}^{L} \dot{y}_{0}(x) X_{n}(x) d x\right\} .
\end{aligned}
$$

From Equation (35) and Equation (36), we obtain the following expression for the acceleration of the points lying in the cross section of the beam along abscissa $x=a$ :

$$
\left.\ddot{y}(x, t)\right|_{x=a}=\sum_{n=1}^{\infty} e^{-\alpha_{n} t}\left(C_{1 n} e^{p_{n}^{*} t}+C_{2 n} e^{-p_{n}^{*} t}\right)=\sum_{n=1}^{\infty} e^{-\alpha_{n} t}(\underbrace{C_{1 n}^{*}}_{C_{1 n}+C_{2 n}} \operatorname{ch}\left(p_{n}^{*} t\right)+\underbrace{C_{2 n}^{*}}_{C_{1 n}-C_{2 n}} \operatorname{sh}\left(p_{n}^{*} t\right)),
$$

where: $C_{1 n}=\left(\alpha_{n}-p_{n}^{*}\right)^{2} C_{1 n}^{\prime}, C_{2 n}=\left(\alpha_{n}+p_{n}^{*}\right)^{2} C_{2 n}^{\prime}$.

\subsection{Second Step}

When damping forces are present, the differential equations, describing the vibrations of the linearized system about the static equilibrium point in Equation (12), are described in the form:

$$
\sum_{j=1}^{2}\left(a_{i j} \ddot{\eta}_{j}+b_{i j} \dot{\eta}+c_{i j} \eta\right)=Q_{i}(t) \quad(i=1,2) \text {. }
$$

The symmetric square matrices of inertial, dissipative, and potential coefficients for the two dynamical models and the matrices of generalized coordinates and forces are denoted, respectively, by:

$$
\mathbf{A}=\left[a_{i j}\right]=\left(\begin{array}{ll}
a_{11} & a_{12} \\
a_{21} & a_{22}
\end{array}\right) ; \mathbf{B}=\left[b_{i j}\right] ; \mathbf{C}=\left[c_{i j}\right] ; \boldsymbol{\eta}=\left[\eta_{i j}\right]=\left[\begin{array}{l}
\eta_{1} \\
\eta_{2}
\end{array}\right] ; \mathbf{Q}(t)=\left[Q_{i}(t)\right] .
$$

Then, the equations of motion from Equation (38) have the following matrix form:

$$
\mathbf{A} \ddot{\boldsymbol{\eta}}+\mathbf{B} \dot{\boldsymbol{\eta}}+\mathbf{C} \boldsymbol{\eta}=\mathbf{Q}(t) .
$$

According to Lagrange's theorem and Sylvester's minorant criterion, the sufficient condition for stability of the equilibrium of the vibrating system is satisfied, i.e. $c_{i i}>0$.

We introduce principal coordinates in vector form:

$$
\boldsymbol{\eta}=\mathbf{H} \mathbf{P},
$$

where $\mathbf{H}=\left[\boldsymbol{\mu}_{1}, \boldsymbol{\mu}_{2}\right]$, whose columns are the normalized eigenvectors, and $\mathbf{P}=\left[\rho_{1}, \rho_{2}\right]^{\mathrm{T}}$ is a vector of principal coordinates.

We substitute Equation (40) in Equation (39), multiply on the left by the matrix $\mathbf{H}^{\mathrm{T}}$, and obtain the matrix equation of motion in the form:

$$
\mathbf{A}^{*} \ddot{\mathbf{P}}+\mathbf{B}^{*} \dot{\mathbf{P}}+\mathbf{C}^{*} \mathbf{P}=\mathbf{F}^{*}
$$

where $\mathbf{A}^{*}=\mathbf{H}^{\mathrm{T}} \mathbf{A} \mathbf{H}, \mathbf{B}^{*}=\mathbf{H}^{\mathrm{T}} \mathbf{B} \mathbf{H}, \mathbf{C}^{*}=\mathbf{H}^{\mathrm{T}} \mathbf{C} \mathbf{H}$ and $\mathbf{F}^{*}=\mathbf{H}^{\mathrm{T}} \mathbf{Q}(t)$.

The expanded form of Equation (41) is:

$$
\bar{a}_{k} \ddot{\rho}_{k}+\bar{b}_{k} \dot{\rho}_{k}+\bar{c}_{k} \rho_{k}=\bar{F}_{k} \quad(k=1,2),
$$

where $\bar{a}_{k}, \bar{b}_{k}, \bar{c}_{k}$ are the diagonal elements of the matrices $\mathbf{A}^{*}, \mathbf{B}^{*}, \mathbf{C}^{*}$ and $\bar{F}_{k}=\sum^{n} \mu_{k j} Q_{j}(t)(n=2)$. The equations in Equation (42) are not coupled and their solutions can be determined for different time-dependencies of $Q_{i}(t)$.

The eigenvectors $\boldsymbol{\mu}_{1}, \boldsymbol{\mu}_{2}$, with respect to the first principal coordinate, have the form: $\boldsymbol{\mu}_{1}=\left[\begin{array}{c}1 \\ \mu_{21}\end{array}\right], \boldsymbol{\mu}_{2}=\left[\begin{array}{c}1 \\ \mu_{22}\end{array}\right]$.

The diagonal elements of the matrices $\mathbf{A}^{*}, \mathbf{B}^{*}, \mathbf{C}^{*}$ are: 


$$
\begin{gathered}
\bar{a}_{1}=a_{11}+\mu_{21}{ }^{2} a_{22}=m_{1}+\mu_{21}{ }^{2} m_{2}, \bar{a}_{2}=a_{11}+\mu_{22}{ }^{2} a_{22}=m_{1}+\mu_{22}{ }^{2} m_{2}, \\
\bar{c}_{1}=c_{11}+2 \mu_{21} c_{12}+\mu_{21}{ }^{2} c_{22}=c_{1}{ }^{*}\left(1+\mu_{21}\right)^{2}+\mu_{21}{ }^{2} c_{2}{ }^{*}, \bar{c}_{2}=c_{11}+2 \mu_{22} c_{12}+\mu_{22}{ }^{2} c_{22}=c_{1}{ }^{*}\left(1+\mu_{22}\right)^{2}+\mu_{22}{ }^{2} c_{2}{ }^{*}, \\
\bar{b}_{1}=c_{b}\left(1+\mu_{21}\right)^{2}+\mu_{21}{ }^{2} c_{a}, \bar{b}_{2}=c_{b}\left(1+\mu_{22}\right)^{2}+\mu_{22}{ }^{2} c_{a},
\end{gathered}
$$

for dynamical model 1

$$
\begin{array}{lll}
\mu_{21}=1-\frac{m_{1}}{c_{1}^{*}} \omega_{1}^{2}, \mu_{22}=1-\frac{m_{1}}{c_{1}^{*}} \omega_{2}^{2}, & ; & \mu_{21}=-1+\frac{m_{1}}{c_{1}^{*}} \omega_{1}^{2}, \mu_{22}=-1+\frac{m_{1}}{c_{1}^{*}} \omega_{2}^{2}, \\
\bar{F}_{1}=Q_{1}+\mu_{21} Q_{2}=\left(m_{1}+\mu_{21} m_{2}\right) \ddot{y}(a, t), & ; & \bar{F}_{1}=\left(m_{1}-\mu_{21} m_{2}\right) \ddot{y}(a, t), \\
\bar{F}_{2}=Q_{1}+\mu_{22} Q_{2}=\left(m_{1}+\mu_{22} m_{2}\right) \ddot{y}(a, t), & ; & \bar{F}_{2}=\left(m_{1}-\mu_{22} m_{2}\right) \ddot{y}(a, t) .
\end{array}
$$

In canonical form, equations from Equation (42) become:

$$
\ddot{\rho}_{k}+2 \bar{\varepsilon}_{k} \dot{\rho}_{k}+\omega_{k}^{2} \rho_{k}=F_{k} \quad(k=1,2),
$$

where $2 \bar{\varepsilon}_{k}=\frac{\bar{b}_{k}}{\bar{a}_{k}}=\frac{c_{b}\left(1+\mu_{2 k}\right)^{2}+\mu_{2 k}{ }^{2} c_{a}}{m_{1}+\mu_{2 k}{ }^{2} m_{2}}, \omega_{k}^{2}=\frac{\bar{c}_{k}}{\bar{a}_{k}}$, and $F_{k}=\frac{\bar{F}_{k}}{\bar{a}_{k}}=\frac{m_{1} \pm \mu_{2 k} m_{2}}{m_{1}+\mu_{2 k}{ }^{2} m_{2}} \ddot{y}(a, t)(+$ for dynamical model 1; - for dynamical model 2).

Taking Equation (36), Equation (37) into account when expressing $F_{k}$, we can express Equation (44) in the following form:

$$
\ddot{\rho}_{k}+2 \bar{\varepsilon}_{k} \dot{\rho}_{k}+\omega_{k}^{2} \rho_{k}=\sum_{n=1}^{\infty} e^{-\alpha_{n} t}\left(M_{n k} e^{p_{n}^{*} t}+N_{n k} e^{-p_{n}^{*} t}\right),
$$

where $M_{n k}=\frac{\left(m_{1} \pm \mu_{2 k} m_{2}\right)}{m_{1}+\mu_{2 k}{ }^{2} m_{2}} C_{1 n} ; N_{n k}=\frac{\left(m_{1} \pm \mu_{2 k} m_{2}\right)}{m_{1}+\mu_{2 k}{ }^{2} m_{2}} C_{2 n}$.

The solution of Equation (45) can be written as a sum of the solution of the corresponding homogeneous equation, obtained with general initial conditions, and the particular solution, obtained with zero initial conditions from Duhamel's integral:

$$
\rho_{k}=e^{-\bar{\varepsilon}_{k} t}\left[\rho_{k, 0} \cos \left(\omega_{k} t\right)+\frac{\dot{\rho}_{k, 0}+\bar{\varepsilon}_{k} \rho_{k, 0}}{\omega_{k}} \sin \left(\omega_{k} t\right)\right]+\frac{1}{\omega_{k}} \int_{0}^{t} \sum_{n=1}^{\infty} e^{-\alpha_{n} \tau}\left(M_{n k} e^{p_{n}^{*} \tau}+N_{n k} e^{-p_{n}^{*} \tau}\right) e^{-\bar{\varepsilon}_{k}(t-\tau)} \sin \omega_{k}(t-\tau) d \tau .
$$

We solve the Duhamel integrals in Equation (46), group the terms, and obtain the following for the principal coordinates $\rho_{k}(t)$ :

$$
\begin{aligned}
\rho_{k}= & \sum_{n=1}^{\infty} e^{-\bar{\varepsilon}_{k} t}\left\{\left[\rho_{k, 0}-\left[\frac{M_{n k}}{\left[\bar{\varepsilon}_{k}-\left(\alpha_{n}-p_{n}^{*}\right)\right]^{2}+\omega_{k}^{2}}+\frac{N_{n k}}{\left[\bar{\varepsilon}_{k}-\left(\alpha_{n}+p_{n}^{*}\right)\right]^{2}+\omega_{k}^{2}}\right]\right] \cos \left(\omega_{k} t\right)+\right. \\
& +\left[\frac{\dot{\rho}_{k, 0}+\bar{\varepsilon}_{k} \rho_{k, 0}}{\omega_{k}}-\frac{1}{\omega_{k}}\left[\frac{M_{n k}\left[\bar{\varepsilon}_{k}-\left(\alpha_{n}-p_{n}^{*}\right)\right]}{\left[\bar{\varepsilon}_{k}-\left(\alpha_{n}-p_{n}^{*}\right)\right]^{2}+\omega_{k}^{2}}+\frac{N_{n k}\left[\bar{\varepsilon}_{k}-\left(\alpha_{n}+p_{n}^{*}\right)\right]}{\left[\bar{\varepsilon}_{k}-\left(\alpha_{n}+p_{n}^{*}\right)\right]^{2}+\omega_{k}^{2}}\right]\right] \sin \left(\omega_{k} t\right)+ \\
& \left.+\frac{M_{n k}}{\left[\bar{\varepsilon}_{k}-\left(\alpha_{n}-p_{n}^{*}\right)\right]^{2}+\omega_{k}^{2}} e^{-\left(\alpha_{n}-p_{n}^{*}\right) t}+\frac{N_{n k}}{\left[\bar{\varepsilon}_{k}-\left(\alpha_{n}+p_{n}^{*}\right)\right]^{2}+\omega_{k}^{2}} e^{-\left(\alpha_{n}+p_{n}^{*}\right) t}\right\} .
\end{aligned}
$$

Using Equation (40), we go back to the original coordinates:

$$
\boldsymbol{\eta}=\left(\begin{array}{l}
\eta_{1} \\
\eta_{2}
\end{array}\right)=\left(\begin{array}{cc}
1 & 1 \\
\mu_{21} & \mu_{22}
\end{array}\right) \cdot\left(\begin{array}{l}
\rho_{1} \\
\rho_{2}
\end{array}\right)=\left(\begin{array}{c}
\rho_{1}+\rho_{2} \\
\mu_{21} \rho_{1}+\mu_{22} \rho_{2}
\end{array}\right),
$$


and from Equation (9) and Equation (48), we determine the initial conditions for the principal coordinates in Equation (47):

$$
t=0: \rho_{1,0}=\frac{\eta_{2,0}-\mu_{22} \eta_{1,0}}{\mu_{21}-\mu_{22}} ; \dot{\rho}_{1,0}=\frac{\dot{\eta}_{2,0}-\mu_{22} \dot{\eta}_{1,0}}{\mu_{21}-\mu_{22}} ; \rho_{2,0}=\frac{\eta_{1,0} \mu_{21}-\eta_{2,0}}{\mu_{21}-\mu_{22}} ; \dot{\rho}_{2,0}=\frac{\dot{\eta}_{1,0} \mu_{21}-\dot{\eta}_{2,0}}{\mu_{21}-\mu_{22}} .
$$

We obtain the solutions to the partial differential equations in Equation (6) and Equation (7), with canonical form from Equation (30), using the same method as in the first step. We substitute the functions $\eta_{2}(t), \dot{\eta}_{2}(t)$ from Equation (29) in Equation (30) and represent $q(x, t)$ via eigenfunction series:

$$
q(x, t)=\sum_{n=1}^{\infty} X_{n}(x) \cdot P_{n}(t),
$$

Then, we seek a solution to the partial differential equation in the form:

$$
y(x, t)=\sum_{n=1}^{\infty} X_{n}(x) \cdot \varphi_{n}(t) .
$$

Using standard techniques, we obtain the following ordinary differential equations:

$$
\ddot{\varphi}_{n}+2 \alpha_{n} \dot{\varphi}_{n}+\bar{p}_{n}^{2} \varphi_{n}=\frac{P_{n}(t)}{\mu} .
$$

Using the results from the first step, we obtained the following for the right-hand side:

$$
\begin{aligned}
\frac{P_{n}(t)}{\mu}= & K_{n}^{*} \mp \frac{c_{2}^{*} \eta_{2} X_{n}(a)}{\mu} \mp \frac{c_{a} \dot{\eta}_{2} X_{n}(a)}{\mu}= \\
& =K_{n}^{*}+e^{-h_{1} t}\left[A_{n, 1}^{(1)} \cos \left(\omega_{1} t\right)+A_{n, 2}^{(1)} \sin \left(\omega_{1} t\right)\right]+e^{-h_{2} t}\left[A_{n, 1}^{(2)} \cos \left(\omega_{2} t\right)+A_{n, 2}^{(2)} \sin \left(\omega_{2} t\right)\right]
\end{aligned}
$$

where we let: $K_{n}^{*}=-\sqrt{\frac{2}{L}}\left[\frac{g L}{\pi n}(1-\cos (\pi n))+\frac{1}{\mu}\left(m g \pm c_{2}{ }^{*} \Delta_{s t, 2}\right) \sin \left(\frac{\pi n}{L} a\right)\right]$,

$$
\begin{aligned}
& A_{n, 1}^{(1)}=\mp \frac{X_{n}(a) A_{21}}{\mu}\left[c_{2}^{*} \sin \alpha_{1}-c_{a}\left(h_{1} \sin \alpha_{1}-\omega_{1} \cos \alpha_{1}\right)\right], A_{n, 2}^{(1)}=\mp \frac{X_{n}(a) A_{21}}{\mu}\left[c_{2}^{*} \cos \alpha_{1}-c_{a}\left(h_{1} \cos \alpha_{1}+\omega_{1} \sin \alpha_{1}\right)\right], \\
& A_{n, 1}^{(2)}=\mp \frac{X_{n}(a) A_{22}}{\mu}\left[c_{2}^{*} \sin \alpha_{2}-c_{a}\left(h_{2} \sin \alpha_{2}-\omega_{2} \cos \alpha_{2}\right)\right], A_{n, 2}^{(2)}=\mp \frac{X_{n}(a) A_{22}}{\mu}\left[c_{2}^{*} \cos \alpha_{2}-c_{a}\left(h_{2} \cos \alpha_{2}+\omega_{2} \sin \alpha_{2}\right)\right],
\end{aligned}
$$

and,$\pm \mp$ correspond to,+- for dynamical model 1 and,-+ for dynamical model 2 .

The solution of equations from Equation (52) can be written as a sum of the solution to the homogeneous equation, obtained with general initial conditions, and the particular solution, obtained with zero initial conditions from Duhamel's integral. The resulting expression, after rearranging terms, is:

$$
\begin{array}{r}
\varphi_{n}=\frac{K_{n}^{*}}{\bar{p}_{n}^{2}}+\frac{1}{\bar{p}_{n}^{2}} e^{-\alpha_{n} t}\left[C_{1 n}^{*} \operatorname{ch}\left(p_{n}^{*} t\right)+C_{2 n}^{*} \operatorname{sh}\left(p_{n}^{*} t\right)\right]+\frac{1}{2 \bar{p}_{n}^{2}} \sum_{k=1}^{2}\left\{e ^ { - h _ { k } t } \left\{\left[\frac{A_{n, 1}^{(k)}\left(\alpha_{n}-h_{k}-p_{n}^{*}\right)-A_{n, 2}^{(k)} \omega_{k}}{\left(\alpha_{n}-h_{k}-p_{n}^{*}\right)^{2}+\omega_{k}^{2}}+\right.\right.\right. \\
\left.+\frac{-A_{n, 1}^{(k)}\left(\alpha_{n}-h_{k}+p_{n}^{*}\right)+A_{n, 2}^{k} \omega_{k}}{\left(\alpha_{n}-h_{k}+p_{n}^{*}\right)^{2}+\omega_{k}^{2}}\right] \cos \left(\omega_{k} t\right)+\left[\frac{A_{n, 1}^{(k)} \omega_{k}+A_{n, 2}^{(k)}\left(\alpha_{n}-h_{k}-p_{n}^{*}\right)}{\left(\alpha_{n}-h_{k}-p_{n}^{*}\right)^{2}+\omega_{k}^{2}}+\right. \\
\left.\left.+\frac{-A_{n, 1}^{(k)} \omega_{k}-A_{n, 2}^{(k)}\left(\alpha_{n}-h_{k}+p_{n}^{*}\right)}{\left(\alpha_{n}-h_{k}+p_{n}^{*}\right)^{2}+\omega_{k}^{2}}\right] \sin \left(\omega_{k} t\right)\right\}+e^{-\left(\alpha_{n}+p_{n}^{*}\right) t} \frac{A_{n, 1}^{(k)}\left(\alpha_{n}-h_{k}+p_{n}^{*}\right)-A_{n, 2}^{(k)} \omega_{k}}{\left(\alpha_{n}-h_{k}+p_{n}^{*}\right)^{2}+\omega_{k}^{2}}+ \\
\left.+e^{-\left(\alpha_{n}-p_{n}^{*}\right) t} \frac{-A_{n, 1}^{(k)}\left(\alpha_{n}-h_{k}-p_{n}^{*}\right)+A_{n, 2}^{k} \omega_{k}}{\left(\alpha_{n}-h_{k}-p_{n}^{*}\right)^{2}+\omega_{k}^{2}}\right\},
\end{array}
$$


where the constants of integration in the initial conditions from Equation (9) are:

$$
C_{1 n}^{*}=\bar{p}_{n}^{2} \int_{0}^{L} y_{0}(x) X_{n}(x) d x-K_{n}^{*}, C_{2 n}^{*}=\frac{\alpha_{n}}{p_{n}^{*}} C_{1 n}^{*}+\frac{\bar{p}_{n}^{2}}{p_{n}^{*}} \int_{0}^{L} \dot{y}_{0}(x) X_{n}(x) d x .
$$

The expressions in Equation (32) and Equation (54) allow us to obtain the solution of partial differential equations in Equation (6) and Equation (7) with initial conditions from Equation (9) in a form determined by the expansion in Equation (51).

\section{Numerical Example}

The results obtained in this work were used to analyze the vibrations of a real mechanical system. The system includes a rope electric hoist T0224 with a lifting capacity of $5 \mathrm{kN}$, lifting height of $12 \mathrm{~m}$, rope diameter $5.7 \mathrm{~mm}$, mass $108 \mathrm{~kg}$, and a single rail consisting of a beam with a cross-section double T-№18M according to GOST 19425-74. The following numerical values were used in the experiment for dynamical model 1 are shown in Table 1.

Table 1. Numerical values of variable

\begin{tabular}{ll}
\hline Beam & $L=5$ \\
\hline Length (m) & $E=2.10^{11}$ \\
Elastic module (Pa) & $J=1760.10^{-8}$ \\
Second moment of area $\left(\mathrm{m}^{4}\right)$ & $\mu=25.8$ \\
Mass per unit length $(\mathrm{kg})$ & $k_{0}=2.5 .10^{10}$ \\
Viscoelastic parameter $\left(\mathrm{Nsm}^{-2}\right)$ & \\
Point mass & $m=48$ \\
Mass (kg) & $a=1$ \\
Distance from support (m) & \\
First load and its elastic rope & $m_{2}^{\prime}=108$ \\
Mass of first load (kg) & $c_{2}=279000$ \\
Stiffness (Nm $\left.{ }^{-1}\right)$ & $\gamma_{2}=0.2$ \\
Small parameter & $p_{2}=0.61$ \\
Linear density (kgm $\left.{ }^{-1}\right)$ & $l_{0,2}=1$ \\
Free length (m) & \\
Suspended load and its elastic rope & \\
Mass of suspended load (kg) & $m_{1}^{\prime}=500$ \\
Stiffness (Nm $\left.{ }^{-1}\right)$ & $c_{1}=93124$ \\
Small parameter & $\gamma_{1}=0.22$ \\
Linear density (kgm $\left.{ }^{-1}\right)$ & $p_{1}=0.095$ \\
Free length (m) & $l_{0,1}=10$ \\
\hline & \\
\hline
\end{tabular}

The numerical simulation was based on our analytical results and was carried out with initial conditions corresponding to one of the typical regimes of operation of suspended load systems. The load with mass $m_{1}^{\prime}=500 \mathrm{~kg}$, suspended at a distance $a=1 \mathrm{~m}$, is descending at a constant velocity $v=\dot{\eta}_{1,0}=8 \mathrm{~m} / \mathrm{min}=0.133 \mathrm{~m} / \mathrm{s}$ and then its motion is suddenly stopped. The transverse motion $y(x=1, t)$ obtained from the first natural frequency and the shape of the first mode are shown in Figure 7 and Figure 8, respectively. The changes in the spring lengths $\eta_{1}(t), \eta_{2}(t)$ with respect to static equilibrium levels are shown in Figure 9 - Figure 12. Figure 7, Figure 9, and Figure 11 show results obtained with small values for the viscous damping coefficients $c_{a}, c_{b}$, while Figure 8, Figure 10, and Figure 12 show results obtain with higher values. 


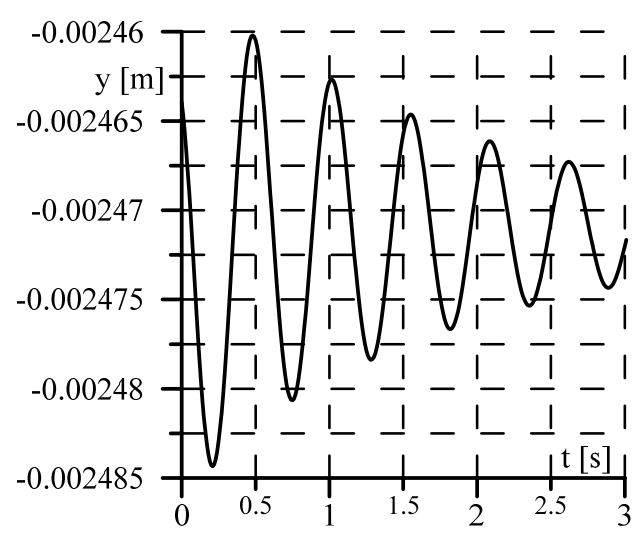

Figure 7. Transverse motion $y=y(x, t)$ at a distance $x=a$ where $c_{a}=1000 \mathrm{Ns} \mathrm{m}^{-1}, c_{b}=800 \mathrm{Ns} \mathrm{m}^{-1}$

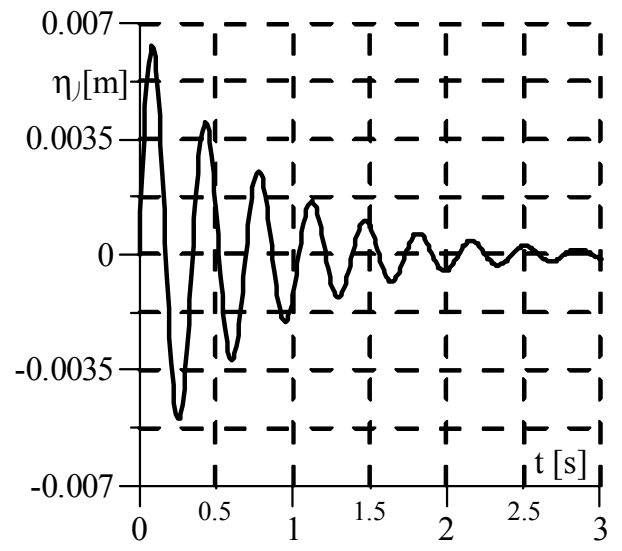

Figure 9. Change in the spring length $\eta_{1}=\eta_{1}(t)$ where $c_{a}=1000 \mathrm{Ns} \mathrm{m}^{-1}, c_{b}=800 \mathrm{Ns} \mathrm{m}^{-1}$

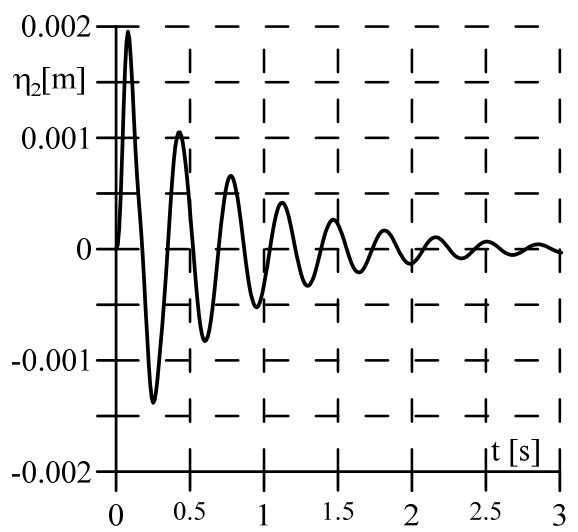

Figure 11. Change in the spring length $\eta_{2}=\eta_{2}(t)$ where $c_{a}=1000 \mathrm{Ns} \mathrm{m}^{-1}, c_{b}=800 \mathrm{Ns} \mathrm{m}^{-1}$

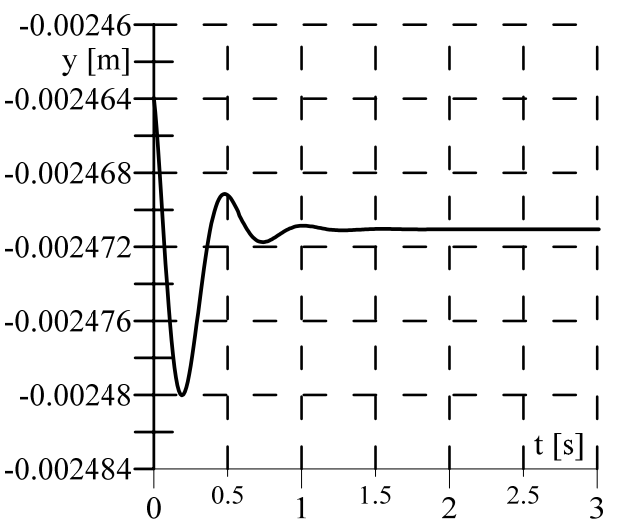

Figure 8. Transverse motion $y=y(x, t)$ at a distance $x=a$ where $c_{a}=1600 \mathrm{Ns} \mathrm{m}^{-1}, c_{b}=8000 \mathrm{Ns} \mathrm{m}^{-1}$

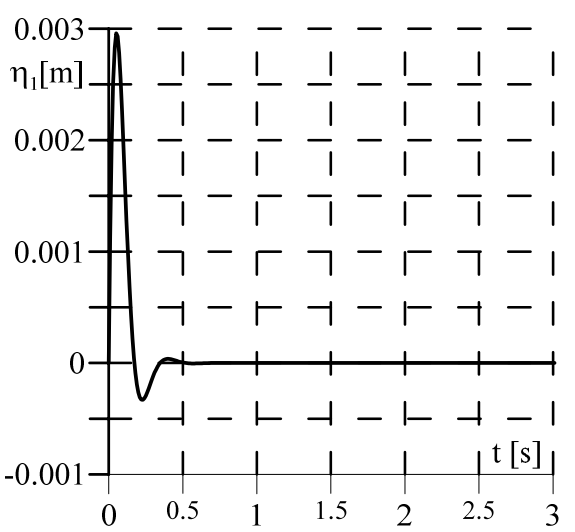

Figure 10. Change in the spring length $\eta_{1}=\eta_{1}(t)$ where $c_{a}=1600 \mathrm{Ns} \mathrm{m}^{-1}, c_{b}=8000 \mathrm{Ns} \mathrm{m}^{-1}$

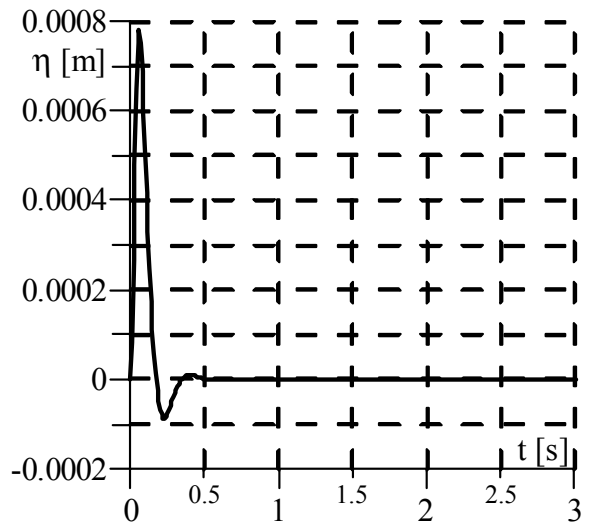

Figure 12. Change in the spring length $\eta_{2}=\eta_{2}(t)$ where $c_{a}=1600 \mathrm{Ns} \mathrm{m}^{-1}, c_{b}=8000 \mathrm{Ns} \mathrm{m}^{-1}$

The elastic force $F_{s p}$, obtained using the nonlinear spring model with stiffness coefficients $c_{1}, c_{3}$ and linearized model with stiffness coefficients $c_{1}^{*}$ is shown in Figure 13. We observe a very good agreement between the simulation and the analytical results which is visible from the coincidence of the schedules.

The numerical simulation was programmed in Visual Fortran. 


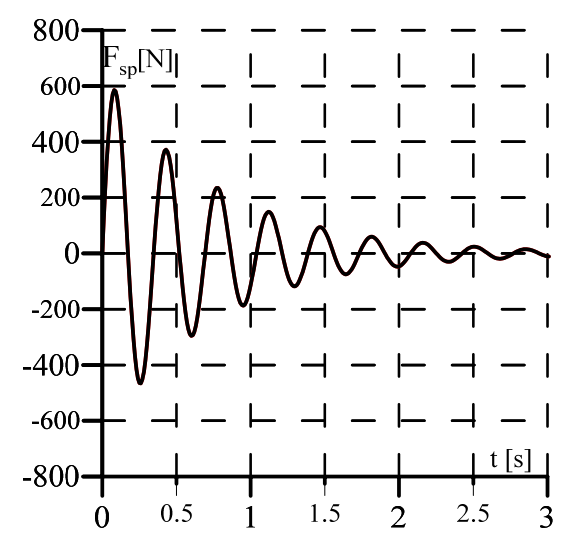

Figure 13. Elastic force $F_{s p}=F_{s p}(t)$ in a main elastic rope

The analytical results enable us to determine and analyze the dynamic load on the different elements in the dynamical model and in turn on the elements of the suspended load system. The approach used to solve the system of differential equations of motion is sufficiently accurate for the intended applications (Zlatanov, Buchvarov, \& Atanasova, 2012). Moreover, the proposed approach allows us to repeat the two solution steps to confirm or increase the accuracy of the results. For example, based on the numerical simulation it is possible to specify more

precise values for $A_{m, i}(i=1,2)$ in (11). Similarly, a specific value for $m_{1} \ddot{y}(a, t)=\left.m_{1} \frac{\partial^{2} y(x, t)}{\partial t^{2}}\right|_{\substack{t=0 \\ x=a}}$ can be used during the derivation of $Q_{i}(i=1,2)$ in Equation (12). In this case, it would be necessary to add a particular solution to the solution of Equation (22), which considering the right-hand side of the system, would consist of constants and can be determined from the initial conditions.

\section{Conclusions}

This paper considered a mechanical system composed of a load, twice elastically suspended from an elastic beam via another load. The following conclusions can be made:

1) Two dynamical models of the mechanical system, which respect the kinetic energy of the elastic ropes, were built. The corresponding differential equations of motion were obtained. The vibration of the mechanical system was described by a coupled system of two ordinary and $n(n=1,2, \ldots)$ partial differential equations;

2) The nonlinear restoring forces were linearized by the method of equivalent linearization;

3) General representation of the system of differential equations and their analytical solution using general initial conditions was obtained. The constants of integration were determined analytically for a specific instance of the initial conditions, which reflects an important practical case;

4) The mechanical system was simulated numerically with initial conditions corresponding to the typical regimes of operation of real systems with suspended loads;

5) Our simulation and analytical results can be used to investigate the dynamic loading on the different elements in the dynamical model, and in turn on the elements of the mechanical systems with suspended loads.

The analytical solution of the system of differential equations can be used in different areas of the engineering practice as they are necessary for performing qualitative dynamic analysis and for solving the dynamic synthesis problem.

\section{References}

Andrianov, I., V., Danishevs'ky, V., \& Ivankov, A. (2010). Asymptotic methods in the theory of vibrations of beams and plates. Dnepropetrovsk: PDABA.

Babakov, I. (2004). Theory of vibrations. Moscow: Drofa.

Bojaddjiev, G., \& Butschvarov, S. (1967). Assymtotische Einfrequenzschwingungen Eines Elastischen Mathematischen Pendels Mit Beweglichen Auflagen. Ann. of VTUS Applied Mechanics, IV(B.II), 69-82. 
Buchvarov, S., Tcherneva-Popova, Z., \& Banov, S. (1998). Vibration, vibroprotection and noiseprotection of machines. Sofia: St. Kliment Ohridski University Press.

Butenin, N., Lunts, Y., \& Merkin, D. (1985). Course of theoretical mechanics. Moscow: Nauka.

Butschvarov, S. (1970). Assymtotische Einfrequenzschwingungen Eines Elastischen Mathematischen Pendels Mit Beweglichen Auflagen Beim Resonanz. Ann. of VTUS Applied Mechanics, IV(B.II), 135-143.

De Silva, C. W. (2000). Vibration: fundamentals and practice. CRC Press. http://dx.doi.org/10.1201/NOE08493 18085

Den Hartog, J. P. (1956). Mechanical vibrations (5th ed.). New York, NY: McGraw-Hill.

Fryba, L. (1999). Vibration of solid and structures under moving loads (3th ed.). London: Thomas Telford House. http://dx.doi.org/10.1680/vosasuml.35393

Ilin, M., M., Kolesnikov, K., \& Saratov, J. (2001). Theory of vibrations. Moscow: MSTU Bauman.

Kostin, G., \& Saurin, V. (2007). An asymptotic approach to the problem of the free oscillations of a beam. Journal of Applied Mathematics and Mechanics, 70(4), 611-621. http://dx.doi.org/10.1016/j.jappmathmech.2007. 09.014

Lurie, A. (2001). Analytical Mechanics. Berlin: Springer. http://dx.doi.org/10.1007/978-3-540-45677-3

Martikka, H., \& Pollanen, I. (2014). Beam dynamics design using analytical methods for optimizing heat transfer platens dynamics behavior. Mechanical Engineering Research, 4(1), 12-26. http://dx.doi.org/10.5539/mer. $\mathrm{v} 4 \mathrm{n} 1 \mathrm{p} 12$

Ouyang, H., \& Mottershead, J. E. (2007). A numerical-analytical combined method for vibration of a beam excited by a moving flexible body. International journal for numerical method in engineering, 72(10), 1181-1191. http://dx.doi.org/10.1002/nme.2052

Panavko, J. (1976). Basses of the applied theory of the vibrations and blow. Leningrad (St.Peterburg): Mechanical engineering (Machinostronie).

Popov, I. (2012). Theoretical investigation and modeling of polymer concrete composites damping. Part I. Harmonic excitation when using one-mass model. Fundamental Sciences and Applications, Technical University-Sofia, Plovdiv Branches, 18, 59-66.

Prokic, A., Besevic, M., \& Lukic, D. (2014). A numerical method for free vibration analysis of beams. Latin American Journal of Solid and Structures, 11(8), 1432-1444. Revised from: http://www.scielo.br/pdf/lajss/ v11n8/09.pdf

Stepanov, A.(1999). Machine Dynamics. Ekaterinburg: UrORAN.

Taylor, R. L., Fillipou, F. C., Saritas, A., \& Arricchio, F. (2003). A mixed finite element method for beam and frame problems. Computation Mechanics, 31(1-2), 192-203. http://dx.doi.org/10.1007/s00466-003-0410-y

Timoshenko, S., Young, D., \& Weaver, Jr. W. (1974). Vibration problems in engineering. New York, NY: John Wiley \& Sons, Inc.

Zlatanov, V., Buchvarov, S., \& Atanasova, S. (2012). Vibration of a load elastic suspended for elastic beam through another load. Machine Design, 4(1), 33-42. Revised from: http://www.mdesign.ftn.uns.ac.rs/pdf/ 2012/no1/033-042.pdf

Zvang, J., \& Zheng, G. T. (2010). Vibration analysis of an elastic beam subjected to a moving beam with flexible connection. Journal of Engineering Mechanics-ASCE, 136(1), 120-131. http://dx.doi.org/10.1061/ (ASCE)EM.1943-7889.0000063

\section{Copyrights}

Copyright for this article is retained by the author(s), with first publication rights granted to the journal.

This is an open-access article distributed under the terms and conditions of the Creative Commons Attribution license (http://creativecommons.org/licenses/by/3.0/). 